\title{
Piezoresistor Sensor Fabrication by Direct Laser Writing on Hydrogenated Amorphous Silicon
}

\author{
P. Alpuim ${ }^{1,2}$, M.F. Cerqueira ${ }^{1}$, J. Noh ${ }^{2}$, J. Gaspar ${ }^{2}$, and J. Borme ${ }^{2}$ \\ ${ }^{1}$ Department of Physics, Universidade do Minho, Campus de Gualtar, 4715-057 Braga, Portugal. \\ ${ }^{2}$ INL - International Iberian Nanotechnology Laboratory, Av. Mestre José Veiga, 4715-330 \\ Braga, Portugal.
}

\begin{abstract}
In this paper we report on the $532 \mathrm{~nm} \mathrm{Nd:YAG} \mathrm{laser-induced} \mathrm{crystallization} \mathrm{of} 10 \mathrm{~nm}$ thick boron-doped hydrogenated amorphous silicon thin films deposited on flexible polyimide and on rigid oxidized silicon wafers by hot-wire chemical vapor deposition. The dark conductivity increased from $\sim 10^{-7} \Omega^{-1} \mathrm{~cm}^{-1}$, in the as-deposited films, to $\sim 10$ and $50 \Omega^{-1} \mathrm{~cm}^{-1}$ after laser irradiation, on rigid and flexible substrates, respectively. Depending on type of substrate, laser power and fluence, a Raman crystalline fraction between 55 and $90 \%$ was measured in HWCVD films, which was higher than observed in rf-PECVD films (35 - 55\%). Crystallite size remained small in all cases, in the range 6-8 $\mathrm{nm}$. Due to a very high conductivity contrast (>7 orders of magnitude) between amorphous and crystallized regions, it was possible to define conductive paths in the a-Si:H matrix, by mounting the sample on a X-Y software-controlled movable stage under the laser beam, with no need for the usual lithography steps. The resistors scribed by direct laser writing had piezoresistive properties, with positive gauge factor $\sim 1$. The details of the laser interaction process with the Si film were revealed by scanning electron microscopy imaging.
\end{abstract}

\section{INTRODUCTION}

The possibility of direct writing thin semiconductive channels or structures on an insulating substrate, in a photoresist-free process, is attractive for its simplicity, cost effectiveness and wide choice of substrates allowed. A broad range of applications, such as touch screens, flexible displays, various types of sensors and many other large-area electronic devices could benefit from such a process [1]. In this paper, we propose a route to attain that goal, using amorphous silicon (a-Si:H) thin-film technology. The fabricated devices are piezoresistive strain sensors made of B-doped nanocrystalline $\mathrm{Si}$ (nc-Si:H).

When a-Si:H thin films with less than $\sim 50 \mathrm{~nm}$ are deposited on an insulating substrate their electrical conductivity is normally very low $\left(\leq 10^{-7} \Omega^{-1} \mathrm{~cm}^{-1}\right)$ [2]. Adding P or B precursors to the reactive gas mixture during deposition does not lead to an increase in the conductivity, due to a very low doping efficiency in a-Si:H and to a large defect concentration caused by lattice mismatch between film and substrate [3,4]. Upon laser exposure, the film electrical conductivity increases many orders of magnitude, up to $\sim 100 \Omega^{-1} \mathrm{~cm}^{-1}$. This increase is a consequence of laser-induced crystallization of the amorphous tissue accompanied by dopant activation of the impurity atoms $[5,6]$. The dark conductivity contrast between amorphous and crystallized regions is so high (>7 orders of magnitude) that it is possible to define crystallized conductive paths in the a-Si:H substrate, with no need to remove the un-exposed a-Si:H. Using optimized conditions in each case, the sheet resistance of the $10 \mathrm{~nm}$ thick conductive channels is $\sim 100 \mathrm{k} \Omega_{\square}$ for resistors scribed on polyimide substrates and $\sim 1000 \mathrm{k} \Omega_{\square}$ for those scribed on oxidized $\mathrm{Si}$ 
wafer substrates. For comparison, the sheet resistance of a copper track with the same dimensions would be $34 \mathrm{k} \Omega_{\square}$ at RT. With an appropriate choice of the length over width ratio it is possible to use the nanocrystalline Si doped channels as interconnects in low-power, high impedance sensing devices fabricated on plastics and other large-area electronic devices. The highly resistive amorphous Si layer could be used as a passivation layer.

The nanocrystalline silicon resistors show, moreover, piezoresistive response, i.e. their resistance changes under applied strain. The gauge factor, relating relative resistance change to applied strain, is $\sim+1$. The piezoresistance of doped nc-Si:H films with a thickness in the range 100-150 nm and dark conductivity, $\sigma_{\mathrm{d}} \sim 5-10 \Omega^{-1} \mathrm{~cm}^{-1}$ is characterized by a gauge factor $\sim \pm 30$ (positive for $\mathrm{p}$ - and negative for n-type films) [7]. Therefore, there is a large margin for progress regarding the piezoresistive response of the laser crystallized $10 \mathrm{~nm}$ thin piezoresistors, although it is seen that the sign of the gauge factor is preserved, at least in B-doped films.

\section{EXPERIMENT}

B-doped a-Si:H films with thickness $10 \mathrm{~nm}$ were deposited by hot-wire chemical vapor deposition (HW-CVD or HW) on plastic (125 $\mu \mathrm{m}$ thick polyimide, PI) and on $300 \mathrm{~nm} \mathrm{SiO} 2$ on $\mathrm{Si}$ wafers. Similar films were deposited by radio-frequency plasma-enhanced CVD (rf-PECVD or $\mathrm{RF}$ ) on the same types of substrates, for comparison. The films were deposited in an ultrahighvacuum system described elsewhere [4] at a substrate temperature, $T_{\text {sub }}=170{ }^{\circ} \mathrm{C}$. Silane, hydrogen and diborane were used as reactant gases. Hydrogen dilution, $D_{\mathrm{H}}$, is defined as the $\mathrm{H}_{2}$ flow divided by the total gas flow. The $\mathrm{B}_{2} \mathrm{H}_{6}$ to $\mathrm{SiH}_{4}$ gas flow ratio was kept constant at $2 \%$ for all depositions. HW depositions lasted $10 \mathrm{~s}, 15 \mathrm{~s}$ and $40 \mathrm{~s}$ for $D_{\mathrm{H}}=60,80$ and $90 \%$, respectively. A single coiled Ta filament was resistively heated with a DC power supply to the temperature, $T_{\text {fil }}=1750{ }^{\circ} \mathrm{C}$ and the working pressure was kept constant at 40 mTorr. For the RF deposition, the inter-electrode distance was $3 \mathrm{~cm}$, the density of RF power was $350 \mathrm{~mW} / \mathrm{cm}^{2}$, and the pressure was 140 mTorr. Deposition conditions for both HW and RF films are summarized in table I.

Table I - Parameters used for HW and RF CVD depositions

\begin{tabular}{lcc}
\hline Parameter & HW-CVD & rf-PECVD \\
\hline Substrate temperature, $T_{\text {sub }}$ & $170^{\circ} \mathrm{C}$ & $170^{\circ} \mathrm{C}$ \\
Working pressure, $p_{\mathrm{w}}$ & $40 \mathrm{mTorr}$ & $140 \mathrm{mT}$ orr \\
Filament temperature / rf-power, $T_{\text {fil }} / P_{\mathrm{RF}}$ & $1750{ }^{\circ} \mathrm{C}$ & $350 \mathrm{mWcm}^{-2}$ \\
Substrate-to-filament (-rf-electrode) distance, $d_{\text {s-fil(elec) }}$ & $7 \mathrm{~cm}$ & $3 \mathrm{~cm}$ \\
Hydrogen dilution, $D_{\mathrm{H}}$ & $60-90 \%$ & $90-97 \%$ \\
\hline
\end{tabular}

Using the Nd:YAG frequency-doubled $532 \mathrm{~nm}$ laser line of a Witec Raman alpha300M+ confocal system, equipped with a motorized sample positioning XY-stage with a positioning accuracy better than $1 \mu \mathrm{m}$, the films were laser-irradiated in a rectangular pattern defined with the geometry tool of the Witec Control software. Typically, a patterned area of $200 \times 40 \mu \mathrm{m}^{2}$ (or $\left.100 \times 40 \mu \mathrm{m}^{2}\right)$ consisting of 40 parallel lines each $200 \mu \mathrm{m}(100 \mu \mathrm{m})$ long, was written at a scan speed of $15 \mu \mathrm{m} / \mathrm{s}$ on the a-Si:H layer. Laser power was in the range 5 to $40 \mathrm{~mW}$, depending on 
the type of substrate. This pattern was replicated six times, using a laser power above the crystallization threshold ( $\sim 7$ and $\sim 20 \mathrm{~mW}$ for the films on plastic and glass, respectively), each time with a different angle $\left(0^{\circ}, 30^{\circ}, 60^{\circ}, 90^{\circ}, 120^{\circ}, 135^{\circ}\right)$ relative to the sample edge (fig. 1 -a) $)$. The film structure is studied by Raman spectroscopy in the same setup, before and after patterning, using very low laser power $(<0.5 \mathrm{~mW})$, to ensure that no crystallization is induced at this stage. Raman crystalline fraction, $X_{c}$, and crystallite size is calculated according to reference [8]. Four metallic electrodes are lithographically defined by lift-off on each patterned area and the electrical resistance is measured in a four-probe geometry (see fig.1-b)). The piezoresistive response is studied in four-point bending experiments by monitoring the resistance change while the samples are bent along their minor transverse axis, giving rise to a normal, uniaxial strain applied along the main longitudinal axis.

Figure 1-a) is a top view, taken with a CCD camera coupled to a microscope, of the 6 crystallized areas after laser irradiation of the a-Si:H film on $\mathrm{SiO}_{2} / \mathrm{Si}$ substrate at positions L1C1 up to L3C2, at a scan speed of $15 \mu \mathrm{m} / \mathrm{s}$. Figure 1-b) shows the design of the metal contacts mask for the six nc-Si:H resistors in a four-probe geometry.
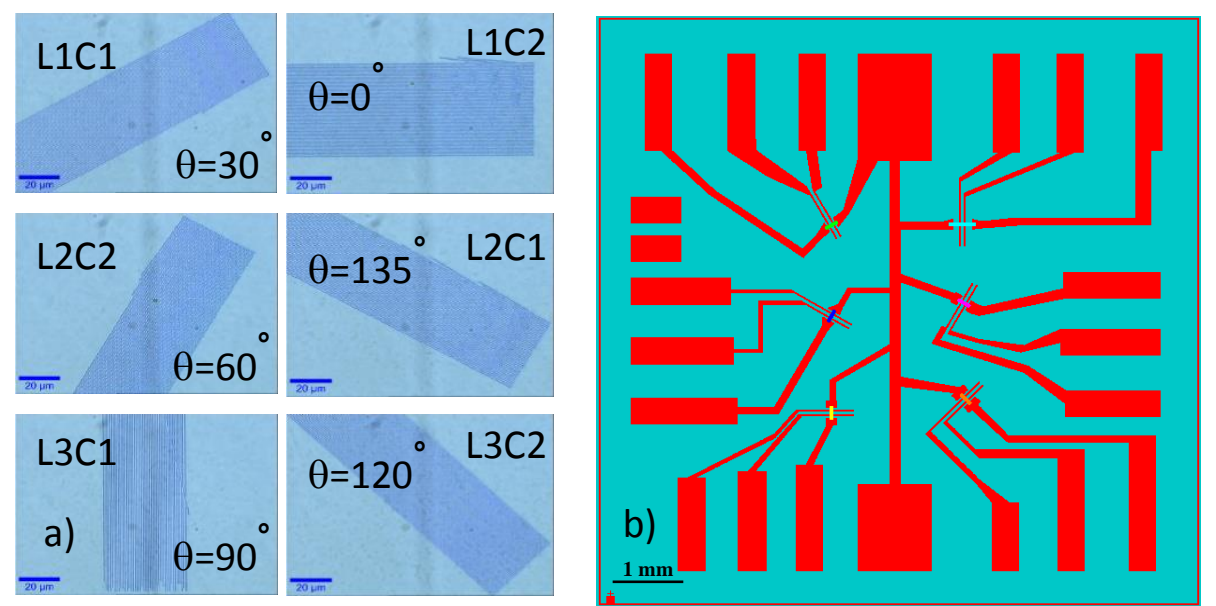

Figure 1. a) Optical micrographs of laser irradiated patterns L1C1 to L3C2 on $10 \mathrm{~nm}$ a-Si:H film on $\mathrm{SiO}_{2} / \mathrm{Si}$ substrate. The rotation angle $\theta$ is measured from the sample edge parallel to stage $\mathrm{X}$-axis. b) Mask design for metal contacts (colors are artificial).

\section{DISCUSSION}

The crystallization threshold for the a-Si:H was studied as a function of the substrate type, CVD deposition technique, and hydrogen dilution of silane. Figure 2-a),b),c) shows the Raman spectra of irradiated areas at a scan speed of $15 \mu \mathrm{m} / \mathrm{s}$ as a function of the laser power used for each exposure. In a) and b), the substrate is $\mathrm{SiO}_{2} / \mathrm{Si}$, whereas in c) it is PI. In a) and c) the a-Si:H deposition was by RF, while in b) it was by HW. Comparing figs.2-a) and b) it can be concluded that the threshold for crystallization is higher for RF films $(\sim 30 \mathrm{~mW})$ than for HW $(\sim 20 \mathrm{~mW})$ and the induced crystallinity is higher in HW films ( $90 \%)$ than in RF ( 40\%). Comparing figures a) and c) it can be seen that amorphous films start crystallizing on plastic substrates at a much lower laser power $(1.5 \mathrm{~mW})$ than on $\mathrm{SiO}_{2} / \mathrm{Si}(30 \mathrm{~mW})$. This is probably due to the very different thermal conductivities of both substrates. Table II shows the initial resistance and conductivity of the a-Si:H film and the resistance, $R$, and $\sigma_{\mathrm{d}}$ of the 6 resistors (L1C1 to L3C2, see also fig.1) for two samples deposited by RF on PI and $\mathrm{Si} / \mathrm{SiO}_{2}$, respectively, using the same $D_{\mathrm{H}}=$ 

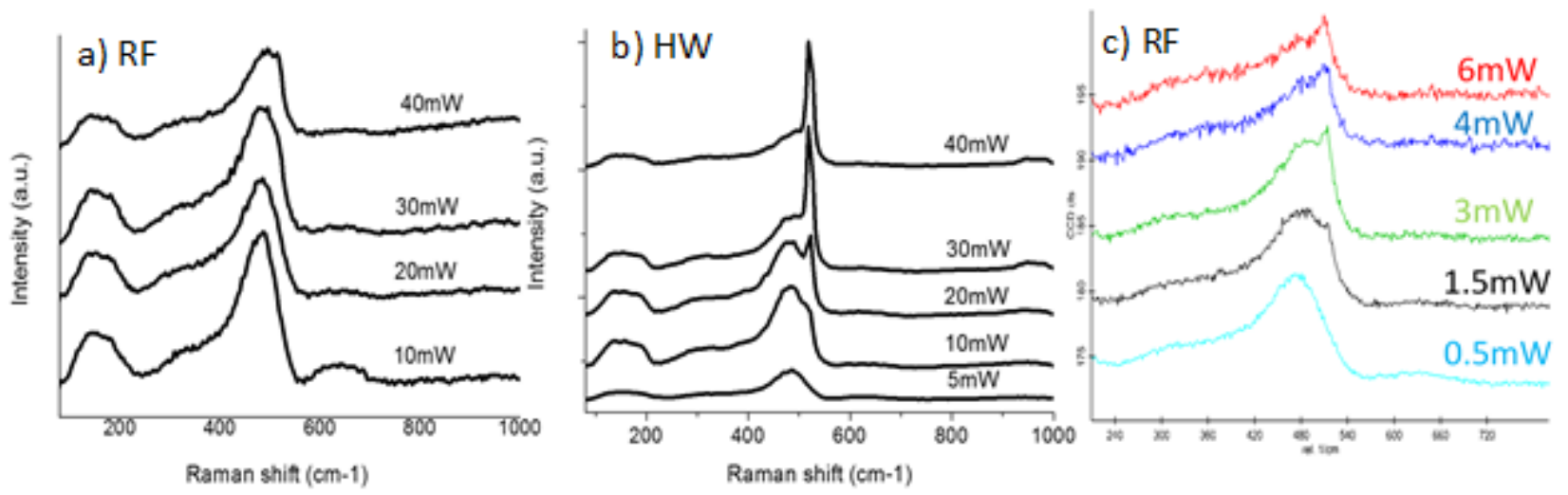

Figure 2. Raman spectra of laser irradiated a) RF films on $\mathrm{SiO}_{2} / \mathrm{Si}$, b) $\mathrm{HW}$ films on $\mathrm{SiO}_{2} / \mathrm{Si}$, c) RF films on polyimide, for different laser power used. Scan speed was $15 \mu \mathrm{m} / \mathrm{s}$ in all cases.

$95 \%$. Laser exposed areas have $\sigma_{\mathrm{d}}$ increased $>7$ (6) orders of magnitude for resistors on PI $\left(\mathrm{SiO}_{2} / \mathrm{Si}\right)$ as compared to $\sigma_{\mathrm{d}}$ of the as-deposited $10 \mathrm{~nm}$ a-Si film. The crystallite size In order to better understand the crystallization process we performed a SEM study of the irradiated areas. Figure 3 shows two such areas where in a) the $40 \mathrm{~mW}$ laser was kept static for approximately $50 \mathrm{~ms}$, and in b) the same laser power was delivered as it moved at a scan speed of $15 \mu \mathrm{m} / \mathrm{s}$. In a) a circular black spot with diameter $80 \mathrm{~nm}$ is centred on the laser spot. It is the most severely heated area where most evaporation took place. Next, there is a circular dark gray area $\varnothing 460 \mathrm{~nm}$ with a non-uniform coverage of recrystallized silicon (only droplets of crystallized Si). A third annular $100 \mathrm{~nm}$ wide region, with lighter contrast, is formed with very

Table II - Resistance, $R$, and dark conductivity, $\sigma_{\mathrm{d}}$, of the irradiated areas at different scanning angle, $\theta$

\begin{tabular}{cccccc}
\hline Position & Scan. Angle & \multicolumn{2}{c}{ Pi substrate } & \multicolumn{2}{c}{ Si/SiO substrate } \\
\hline & $\theta\left(^{\circ}\right)$ & $R(\mathrm{k} \Omega)$ & $\sigma_{\mathrm{d}}\left(\Omega^{-1} \mathrm{~cm}^{-1}\right)$ & $R(\mathrm{k} \Omega)$ & $\sigma_{\mathrm{d}}\left(\Omega^{-1} \mathrm{~cm}^{-1}\right)$ \\
\hline $\mathrm{a}-\mathrm{Si}$ & 0 & $2.5 \times 10^{9}$ & $1 \times 10^{-7}$ & $1.4 \times 10^{8}$ & $2 \times 10^{-6}$ \\
\hline $\mathrm{L} 1 \mathrm{C} 1$ & 30 & 72 & 84 & 39.4 & 63.5 \\
$\mathrm{~L} 1 \mathrm{C} 2 *$ & 0 & 74 & 47 & na & na \\
$\mathrm{L} 2 \mathrm{C} 1$ & 135 & 79 & 76 & 54.5 & 45.9 \\
$\mathrm{~L} 2 \mathrm{C} 2$ & 60 & 93 & 65 & 69.3 & 36.1 \\
$\mathrm{~L} 3 \mathrm{C} 1$ & 90 & 92 & 54 & 44.5 & 56.2 \\
$\mathrm{~L} 3 \mathrm{C} 2$ & 120 & 72 & 84 & 16.6 & 151 \\
\hline \multicolumn{2}{r}{$*$ Resistor L1C2 has twice the irradiated area of the others $\left(400\right.$ or $\left.200 \times 40 \mathrm{~mm}^{2}\right)$}
\end{tabular}

fine grain. This is the area of accumulation where the melted silicon from under the beam reaches and solidifies at. In the remaining part of the figure there is solid phase crystallized silicon, with crystallite size larger than in the previous area but still of nanometric size $(6-8 \mathrm{~nm}$ measured by Raman). The diameter of the laser crystallized area is $1.1 \mu \mathrm{m}$ as estimated from the micrograph shown in the inset of figure 3.-a). Figure 3.-b) shows that, as seen for the single shot case, the centre of the first shot exposed kinetically is depleted of silicon (area B). Meltedsolidified Si accumulates on all sides of the first shot (area A). At a subsequent movement the 

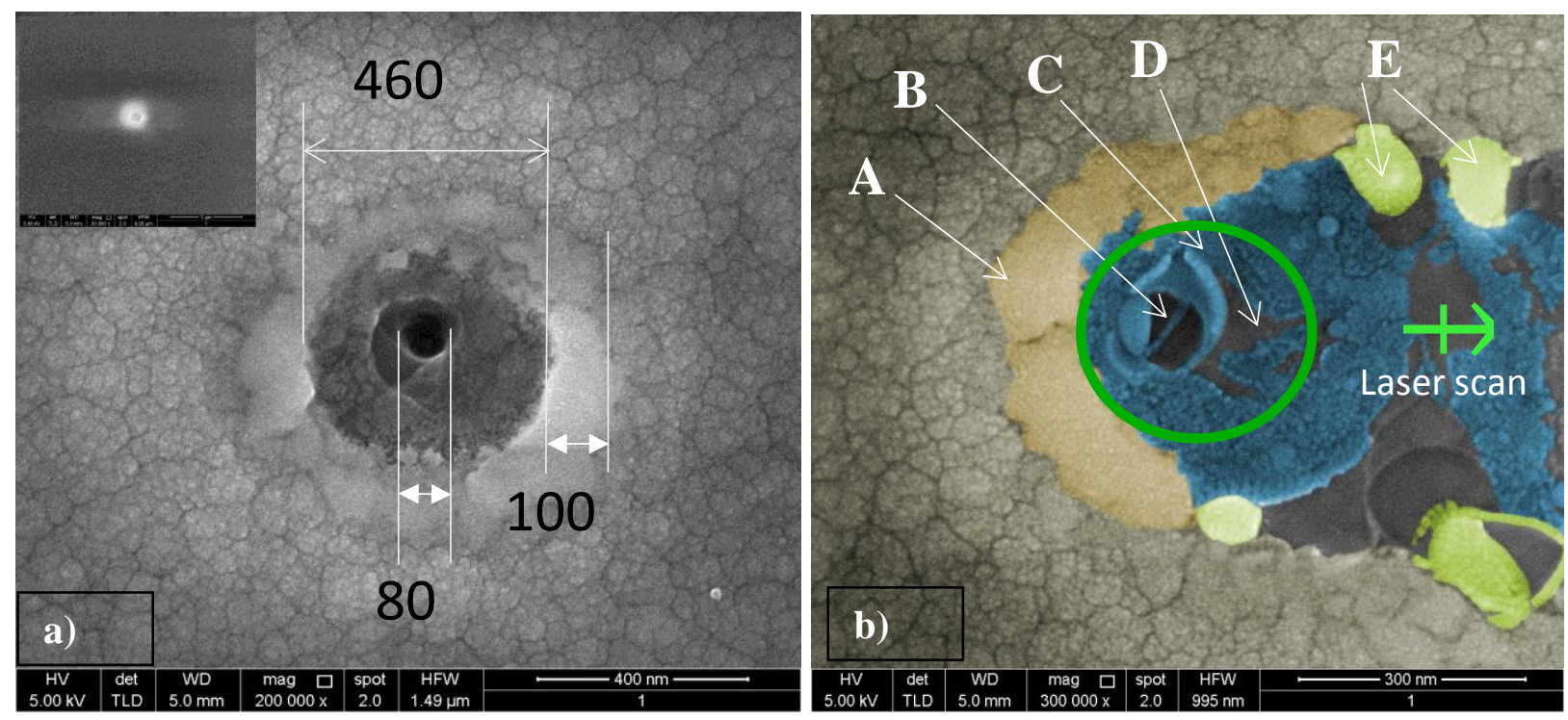

Figure 3 - SEM images of irradiated areas where in a) the $40 \mathrm{~mW}$ laser was kept static for 50 $\mathrm{ms}$, and in b) the same laser power was delivered as it moved at a scan speed of $15 \mu \mathrm{m} / \mathrm{s}$. The letters in b) refer to areas of different laser-Si film interaction, as explained in the text. The colors are not real. The inset in a) shows that total interaction area between the static laser and the film was $1.1 \mu \mathrm{m}$.

laser heats the area of accumulation on one side of the first shot in the direction of the laser scan (C). The accumulated material is still hot and possibly does not have good adhesion to the substrate (D), so it melts immediately and reorganizes in droplets neighboured by areas with no silicon (E). The remaining area shows, like in a), solid phase laterally crystallized silicon. From the SEM study (figure 3 and others not shown) it becomes evident that melted/solidified lines follow the movement and shape of the laser beam and there is etching of the Si film under the laser path which locally decreases the conductance. These areas correspond to $\sim 40 \%$ of the device area. Therefore the effective width of the patterned resistors is less than the apparent width. By changing exposure conditions: decreasing the laser power, increasing the scan speed or increasing the laser lines inter-distance, it may be possible to reduce the width of the track etched by the laser. This would lead to a higher conductance of the devices.

Figure 4-a) shows $\sigma_{d}$ of the crystallized areas as a function of hydrogen dilution used for the deposition of the precursor a-Si:H film. Both for PI and for $\mathrm{SiO}_{2} / \mathrm{Si}$ substrates it is clear that the a-Si:H conditions that yield the higher $\sigma_{\mathrm{d}}$ after irradiation are those corresponding to a $D_{\mathrm{H}}$ at the edge of the amorphous-to-microcrystalline transition that occurs in films with thickness higher than $\sim 100 \mathrm{~nm}: D_{\mathrm{H}}=60$ or $80 \%$ for $\mathrm{HW}$ films on $\mathrm{SiO}_{2}$ and $\mathrm{PI}$, respectively, and $D_{\mathrm{H}}=95 \%$ for RF films deposited on both types of substrates.

Figure 4-b) shows the relative resistance change of crystallized resistors L1C1 and L1C2 defined on a film deposited by RF at $D_{\mathrm{H}}=95 \%$ as a function of strain, $\varepsilon$, obtained from 4-point bending tests. The films show a positive gauge factor, $G F=+1$. The positive $G F$ is in agreement with what is found in thicker B-doped nc-Si:H films deposited by HW and RF [7]. The measurement here is very noisy ( $r=0.87$ ) because of a poor design of the wire connections to the resistors. This will be improved in the short term and GFs will be re-measured and reported in future work. 

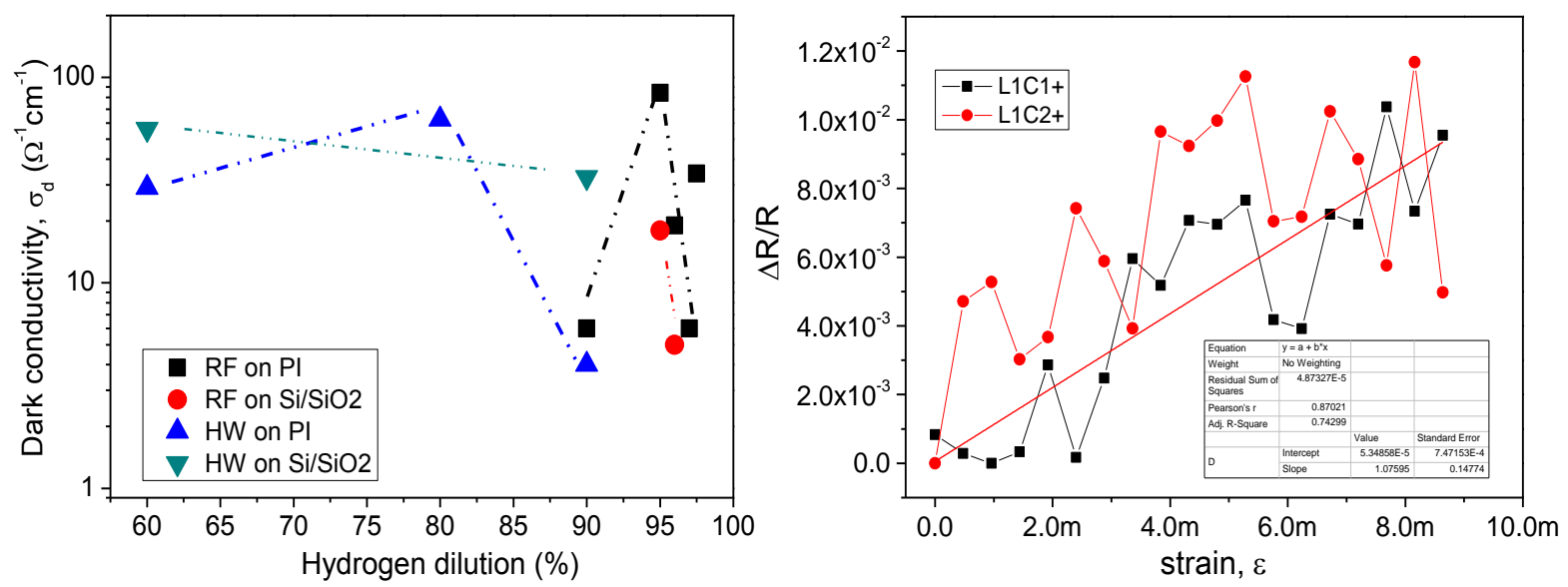

Figure $4-$ a) $\sigma_{\mathrm{d}}$ of the crystallized areas as a function of $D_{\mathrm{H}}$ used for the deposition of the a-Si precursor by $\mathrm{HW}$ (triangles) and $\mathrm{RF}$ (squares and circles) on $\mathrm{PI}$ and $\mathrm{SiO}_{2} / \mathrm{Si}$ substrates; b) relative resistance change as a function of applied strain for resistors L1C1 (squares) and L1C2 (circles) on PI. The straight line is a least squares adjust to $\mathrm{L} 1 \mathrm{C} 1 \mathrm{data}$. The parameters of the linear regression are given in the table inset.

\section{CONCLUSIONS}

Boron doped nc-Si:H structures with high dark conductivity were obtained by $532 \mathrm{~nm}$ laser irradiation of $10 \mathrm{~nm}$ thick a-Si:H films deposited by HW and RF. The dark conductivity is higher for crystallized structures on polyimide substrates than for those on $\mathrm{SiO}_{2} / \mathrm{Si}$ wafer substrates. The laser power threshold for crystallization is lower by a factor of 20 for films on polyimide substrates as compared to films on $\mathrm{SiO}_{2} / \mathrm{Si}$ substrates. The laser power threshold for crystallization is lower by a factor of $\sim 3$ for films deposited by HW-CVD as compared to films deposited by rf-PECVD. Piezoresistive response of the structures was demonstrated and the measured gauge factors were positive and close to 1 .

\section{REFERENCES}

1. P. Fojtík, K. Dohnalová, T. Mates, J. Stuchlík, I. Gregor, J. Chval, A. Fejfar, J. Kočka and I. Pelant, Phil. Mag. B 82 (17), 1785 (2002)

2. H. V. Nguyen, I. An, R. W. Collins, Y. Lu, M. Wakagi, and C. R. Wronski, Appl. Phys. Lett. 66 (26), 3609 (1995)

3. P. Roca i Cabarrocas, N. Layadi, T. Heitz, B. Drévillon, and I. Solomon, Appl. Phys. Lett. 65 (26), 3335 (1994)

4. S.A. Filonovich, M. Ribeiro, A.G. Rolo, P. Alpuim, Thin Solid Films 516, 576 (2008)

5. Y.-J. Lee, J.-D. Kwon, D.-H. Kim, K.-S. Nam, Y. Jeong, S.-H. Kwon, S.-G. Park, Thin Solid Films 542, 388 (2013)

6. F. Gontad, J.C. Conde, S. Filonovich, M.F. Cerqueira, P. Alpuim, S. Chiussi, Thin Solid Films 536, 147 (2013)

7. P. Alpuim, J. Gaspar, P. Gieschke, C. Ehling, J. Kistner, N. J. Gonçalves, M. I. Vasilevskiy, and O. Paul, J. Appl. Phys. 109, 123717 (2011)

8. M. Vanacek, J. Kocka, J. Strichlik, Z. Kosicek, O. Stika, and A. Triska, Sol. Energy Mater. 8, $411(1983)$ 